\title{
AN ABSTRACT FIXED POINT THEOREM FOR NONEXPANSIVE MAPPINGS
}

\author{
W. A. KIRK $^{1}$
}

\begin{abstract}
A class $\delta$ of subsets of a bounded metric space is said to be normal if each member of $\delta$ contains a nondiametral point. An induction proof is given for the following. Suppose $M$ is a nonempty bounded metric space which contains a class $\delta$ of subsets which is countably compact, normal, stable under arbitrary intersections, and which contains the closed balls in $M$. Then every nonexpansive self-mapping of $M$ has a fixed point.
\end{abstract}

In [7], J. P. Penot presents an abstract version of the writer's fixed point theorem of [3] for nonexpansive mappings. Penot's result is based essentially upon the original line of argument (which uses Zorn's lemma). We show here that a different approach yields the abstract result under even weaker assumptions.

Let $(M, d)$ be a metric space; for a subset $D$ of $M$, let

$$
\begin{aligned}
\delta(D) & =\sup \{d(u, v): u, v \in D\}, \\
r_{u}(D) & =\sup \{d(u, v): v \in D\} \quad(u \in D), \\
r(D) & =\inf \left\{r_{u}(D): u \in D\right\},
\end{aligned}
$$

and

$$
h(D)= \begin{cases}r(D) / \delta(D) & \text { if } \delta(D)>0 \\ 1 & \text { if } \delta(D)=0\end{cases}
$$

Definition [7]. A class $\delta$ of subsets of $M$ is said to be normal if for each $D \in \mathcal{\delta}$, $\delta(D)>0 \Rightarrow h(D) \in(0,1)$. The class $\delta$ is said to be [countably] compact if each [countable] subfamily of $\mathcal{S}$ which has the finite intersection property has nonvoid intersection.

Recall that a mapping $T: M \rightarrow M$ is said to be nonexpansive if $d(T(u), T(v))<$ $d(u, v), u, v \in M$. We use $B(u ; r)$ to denote the closed ball centered at $u \in M$ with radius $r>0$.

TheOREM 1. Let $(M, d)$ be a nonempty bounded metric space and suppose $M$ contains a class $\mathcal{S}$ of subsets which is countably compact, stable under arbitrary intersections, and normal. Suppose further that $\mathcal{S}$ contains the closed balls of $M$. Then every nonexpansive mapping $T$ of $M$ into itself has a fixed point.

Received by the editors August 21, 1980.

1980 Mathematics Subject Classification. Primary 54H25; Secondary 47H10.

Key words and phrases. Fixed point theorem, nonexpansive mappings.

${ }^{1}$ Research supported in part by National Science Foundation grant MCS 8001604. 
The above differs from Penot's result in that countable compactness is assumed rather than compactness. We base our proof upon the following abstraction of a lemma due to Gillespie and Williams [2].

LEMMA. Let $(M, d)$ be a nonempty bounded metric space and let $\mathcal{S}$ be a class of subsets of $M$ which contains the closed balls of $M$ and which is stable under arbitrary intersections. Suppose $T: M \rightarrow M$ is nonexpansive. Then for each $\varepsilon>0$ there exists $a$ nonempty set $M(\varepsilon) \in \mathcal{S}$ such that $T: M(\varepsilon) \rightarrow M(\varepsilon)$ and for which $\delta(M(\Sigma))<$ $(h(M)+\varepsilon) \delta(M)$.

Proof. If $\delta(M)=0$, take $M(\varepsilon)=M$. Otherwise, construct $M(\varepsilon)$ as follows. Let $\rho=(h(M)+\varepsilon) \delta(M)$. By the definition of $h$, the set $\mathcal{C}=\{z \in M: M \subset B(z ; \rho)\}$ is nonempty. Let

$$
\mathscr{F}=\{D \in \mathcal{S}: \mathcal{C} \subset D, T: D \rightarrow D\}
$$

and let $L=\cap \mathscr{F}$. Note that $\mathscr{F} \neq \varnothing$ since $M \in \mathscr{F}$. Also $L \in \mathcal{S}, \mathcal{C} \subset L$, and $T$ : $L \rightarrow L$. Let $A=\mathcal{C} \cup T(L)$. Then $A \subset L$; thus $\operatorname{cov}(A)=\cap\{D: D \in \mathcal{S}, A \subset D\}$ $\subset L$ from which $T(\operatorname{cov}(A)) \subset T(L) \subset A \subset \operatorname{cov}(A)$, proving $\operatorname{cov}(A) \in \mathscr{F}$. Therefore $\operatorname{cov}(A)=L$.

Now let

$$
M(\varepsilon)=\{x \in L: L \subset B(x ; \rho)\} .
$$

Then $\mathcal{C} \subset M(\varepsilon)$, so $M(\varepsilon) \neq \varnothing$. Also if $x \in M(\varepsilon)$, then $T(x) \in L$ and for each $y \in L, d(T(x), T(y))<d(x, y)<\rho$. Furthermore if $z \in \mathcal{C}, d(T(x), z)<\rho$ (because $M \subset B(z ; \rho))$. This proves that $A \subset B(T(x) ; \rho)$ which in turn implies $L=$ $\operatorname{cov}(A) \subset B(T(x) ; \rho)$, i.e., $T: M(\varepsilon) \rightarrow M(\varepsilon)$. Finally,

$$
M(\varepsilon)=\left\{\bigcap_{u \in L} B(u ; \rho)\right\} \cap L .
$$

Thus $M(\varepsilon)$ is the intersection of sets in $\mathcal{S}$; hence $M(\varepsilon) \in \mathcal{S}$. Since obviously $\delta(M(\varepsilon))<\rho$, this completes the proof.

Proof of Theorem 1. Let $\mathfrak{N}=\{D \in \mathcal{S}: D \neq \varnothing, T: D \rightarrow D\}$ and for each $D \in \mathfrak{N}$, let $\delta_{0}(D)=\inf \{\delta(F): F \in \mathfrak{R}, F \subset D\}$. Set $D_{1}=M$, and with $D_{1}, \ldots, D_{n}$ given, select $D_{n+1} \in \mathfrak{N}$ so that $D_{n+1} \subset D_{n}$ and

$$
\delta\left(D_{n+1}\right)<\delta_{0}\left(D_{n}\right)+1 / n \text {. }
$$

Let $C=\cap_{n=1}^{\infty} D_{n}$. Then $C \in \mathcal{S}$ and by countable compactness, $C \neq \varnothing$. Thus $C \in \Re$. By the lemma we now have for each $\varepsilon>0$ and $n \in N$,

$$
\delta(C)-1 / n<\delta\left(D_{n+1}\right)-1 / n<\delta_{0}\left(D_{n}\right)<\delta(C(\varepsilon))<(h(C)+\varepsilon) \delta(C) .
$$

Letting $n \rightarrow \infty$,

$$
\delta(C) \leqslant(h(C)+\varepsilon) \delta(C) .
$$

Since this is true for each $\varepsilon>0$,

$$
\delta(C)<h(C) \delta(C),
$$

and because $\mathcal{S}$ is normal this in turn implies $\delta(C)=0$. Therefore $C=\{x\}$ with $T(x)=x$. 
REMARK 1. A constructive proof of Theorem 1 can be given by using the lemma in conjuction with the above, but with $\varepsilon$ depending on $D$.

REMARK 2. In [1], Fuchssteiner gives another constructive proof of the theorem of [3]. (Also see Lim [5].) Our method seems more direct. Fuchssteiner's aproach invokes a fixed point theorem of Zermelo, and consequently an adaptation of that approach to the present setting would require the assumption of compactness on $\mathcal{S}$ rather than countable compactness.

REMARK 3. Suppose $X$ is a Banach space with $\tau$ any topology on $X$ for which the norm closed balls are $\tau$-closed, and say that a $\tau$-closed convex subset $K$ of $X$ has $\tau$-normal structure if for each bounded $\tau$-closed convex subset $D$ of $K, \delta(D)>0 \Rightarrow$ $h(D) \in(0,1)$. Then the following is an immediate special case of Theorem 1 .

THeOReM 2. Let $K$ be a nonempty bounded $\tau$-closed convex subset of $X$ which has $\tau$-normal structure and which is countably compact in the $\tau$-topology. Suppose $T$ : $K \rightarrow K$ is nonexpansive. Then $T$ has a fixed point in $K$.

Proof. Take $\mathcal{S}$ to be the family of $\tau$-closed convex subsets of $K$ and apply Theorem 1.

If $\tau$ is the weak topology on $X$, the above reduces to the original theorem of [3]. Theorem 2 is also known for $X$ a conjugate space and $\tau$ the weak* topology on $X$ ([4], cf. [6]). However, because of the Eberlein-Smulian Theorem and the Alaoglu Theorem, in neither of these instances does the assumption of countable compactness yield greater generality.

\section{REFERENCES}

1. B. Fuchssteiner, Iterations and fixpoints, Pacific J. Math. 68 (1977), 73-80.

2. A. A. Gillespie and B. B. Williams, Fixed point theorem for nonexpansive mappings on Banach spaces with uniformly normal structure, Applicable Anal. 9 (1979), 121-124.

3. W. A. Kirk, A fixed point theorem for mappings which do not increase distances, Amer. Math. Monthly 72 (1965), 1004-1006.

4. __ Mappings of generalized contractive type, J. Math. Anal. Appl. 32 (1970), 567-572.

5. T. C. Lim, A constructive proof of the infinite version of the Belluce-Kirk theorem, Pacific J. Math. 8 (1979), 467-469.

6. Asymptotic centers and nonexpansive mappings in some conjugate spaces, Pacific J. Math. (to appear).

7. J. P. Penot, Fixed point theorems without convexity, Analyse Non Convex (Pau, 1977), Bull. Soc. Math. France Mém. 60 (1979), 129-152.

Department of Mathematics, Unviersity of Iowa, Iowa CtTy, Iowa 52242. 